\title{
Ureteral Stenosis after Uterine Suspension Using TVM (Transvaginal Mesh)
}

\author{
Hun Shan Pan ${ }^{1,2}$, Wei-Jiun Li ${ }^{1}$, Ma Lee Ko ${ }^{4}$, Yan-Fong Lu ${ }^{1}$, Kenneth Pan ${ }^{3}$, Cristiana Pan ${ }^{3}$, Jier Zen Chang ${ }^{1 *}$ and \\ Lee Wen Huang ${ }^{1,2}$
}

${ }^{1}$ Obstetrics and Gynecology Department, Shin Kong Wu Ho Su Memorial Hospital, Taiwan

${ }^{2} \mathrm{Fu}$ Jen Catholic University, Taiwan

${ }^{3}$ University of British Columbia, Canada

${ }^{4}$ Ranchlands Medical Center, Canada

\begin{abstract}
*Corresponding author: Jier Zen Chang, Obstetrics and Gynecology Department, Shin Kong Wu Ho-Su Memorial Hospital, 111 Shilin, Taipei, Taiwan.
\end{abstract}

Received Date: April 16, 2019

Published Date: April 23, 2019

\section{Introduction}

We report a rare case of ureteral stenosis after sacrospinous suspension. A 64-year-old post-menopausal female, G2P2, complained of a heavy sensation and the feeling of sitting on a ball, especially in the afternoon. Tracing back her previous surgical history, she had undergone a cesarean section and subtotal hysterectomy due to myoma with severe adhesion. A pelvic examination revealed total prolapse of the vaginal cervix with stage III cystocele. A urodynamic study revealed bladder outlet obstruction, and a pad test was $13 \mathrm{~g}$ after restoring the vaginal cervix. Therefore, after explaining the risks and complications

of surgery, Pelvic organ prolapse (POP) surgery including sacrospinous suspension (Upholds), mid-urethral sling surgery (Solyx) and anterior compartment repair was performed. Ten days after surgery, she returned to our hospital due to left costovertebral angle pain plus knocking tenderness. CT showed hydronephrosis without urolithiasis, and antegrade pyelography also showed ureteral stenosis in the lower third (Figure 1). After several attempts at stent insertion had failed, a urologist suggested re-implanting the ureter. The postoperative course was uneventful. Intravenous pyelography was performed 4 months after this surgery, and showed a patent left ureter.
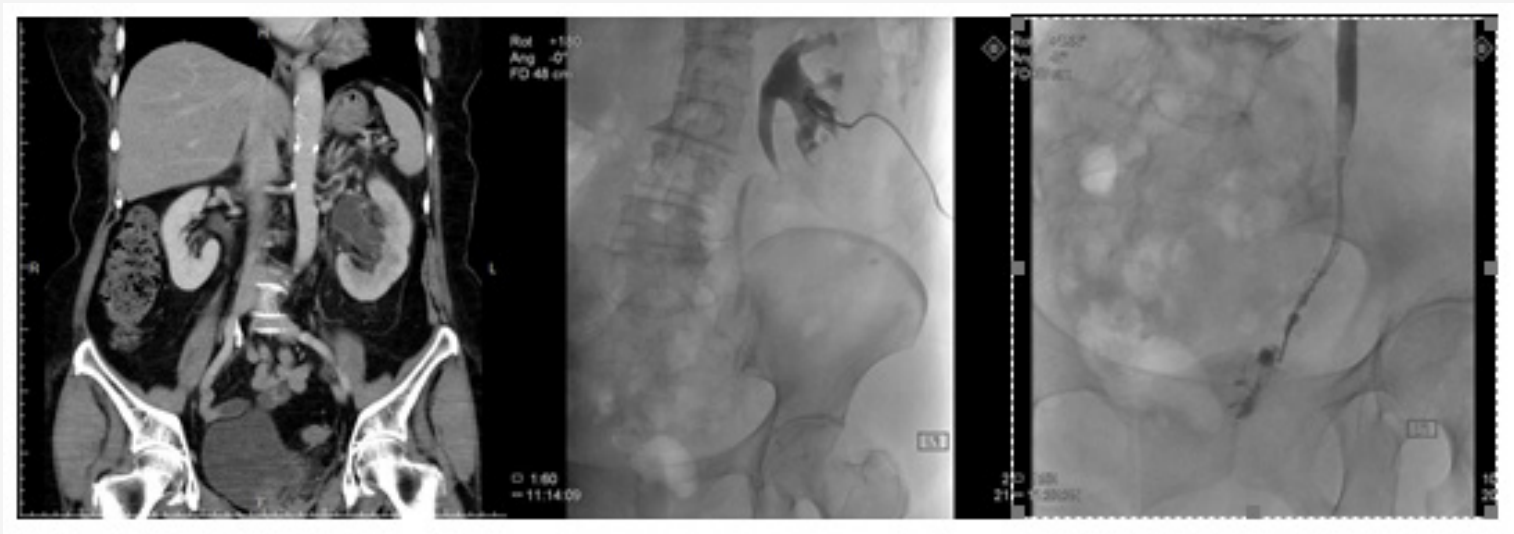

Figure 1: Left hydronephrosis with fatty stranding in the left bladder. Severe stenosis of the left lower ureter with dilatation of the upstream ureter and pelvicalyceal system; a guidewire was passed into the PCN tube and through the ureter to the stenosis site, however it could not pass the stenosis site.

POP is a disabling and chronic condition that affects roughly $20 \%$ of women of all ages both physically and psychologically [1].
Pelvic organs can protrude outside the body through the vagina due to weakness in the pelvic floor, and most women with POP 
also suffer from at least one other pelvic floor disorder such as incontinence. POP increase nationwide, namely, anterior, posterior and middle compartments prolapse. Many different kits have been developed in recent years for reconstructive surgery, among which synthetic material augmentation plays a major role but also results in complications such as dyspareunia and vaginal discomfort.

Surgical mesh can be used for urogynecological procedures, including repair of POP and stress urinary incontinence (SUI). It is implanted to reinforce the weakened vaginal wall for POP repair or support the mid-urethra for the repair of SUI [2]. The most frequent complications include erosion through vaginal epithelium, infection, pain, urinary problems, and recurrence of prolapse and/ or incontinence [3]. There have also been reports of bowel, bladder, and blood vessel perforation during insertion [4]. In some cases, vaginal scarring and mesh erosion have been reported to lead to a significant decrease in patient quality of life due to discomfort and pain, including dyspareunia [5]. POP surgery will be required for patients with total prolapse of the uterus or vault, and we suggest inserting a double $\mathrm{j}$ before surgery in order to prevent ureter kicking subsequent to hydronephrosis. In addition, once the operation has been completed, attempts should be made to loosen the tightness of the mesh around the hanging area with palpitation under a cystoscope to allow the operators to recheck the flow of urine from bilateral ureters. It is mandatory to inform the patients of the risk of such surgery.

\section{Acknowledgement}

None.

\section{Conflict of Interest}

No conflict of interest.

\section{References}

1. Wu JM, Vaughan CP, Goode PS, Redden DT, Burgio KL, et al. (2014) Prevalence and trends of symptomatic pelvic floor disorders in U.S. women. Obstet Gynecol 123(1): 141-148.

2. Lo TS, Wang AC, Liang CC, Long CY, Lee SJ (2006) Treatment for unsuccessful tension-free vaginal tape operation by shortening preimplanted tape. J Urol 175: 2196-2199.

3. Su TH, Lau HH, Huang WC, Hsieh CH, Chang RC, et al. (2014) Singleincision mesh repair versus traditional native tissue repair for pelvic organ prolapse: results of a cohort study. Int Urogynecol J 25(7): 901908.

4. Long CY, Lo TS, Wang CL, Wu CH, Liu CM, et al. (2012) Risk factors of surgical failure following transvaginal mesh repair for the treatment of pelvic organ prolapse. Eur J Obstet Gynecol Reprod Biol 161(2): 224227.

5. Hsieh CH, Hsu CS, Su TH, Chang ST, Lee MC (2007) Risk factors for urinary incontinence in Taiwanese women aged 60 or over. Int Urogynecol J Pelvic Floor Dysfunct 18(11): 1325-1329. 